ARTICLE

Received 17 May 2013 | Accepted 13 Jun 2013 | Published 11 Jul $2013 \quad$ DOl: 10.1038/ncomms3142

\title{
Recognition of vitamin B metabolites by mucosal-associated invariant T cells
}

Onisha Patel ${ }^{1, \star}$, Lars Kjer-Nielsen ${ }^{2, \star}$, Jérôme Le Nours ${ }^{1,3}$, Sidonia B.G. Eckle ${ }^{2}$, Richard Birkinshaw ${ }^{1}$ Travis Beddoe ${ }^{1,3}$, Alexandra J. Corbett ${ }^{2}$, Ligong Liu ${ }^{4}$, John J. Miles ${ }^{5,6}$, Bronwyn Meehan²,

Rangsima Reantragoon², Maria L. Sandoval-Romero', Lucy C. Sullivan², Andrew G. Brooks², Zhenjun Chen², David P. Fairlie ${ }^{4}$, James McCluskey ${ }^{2}$ \& Jamie Rossjohn $n^{1,3,6}$

The mucosal-associated invariant T-cell antigen receptor (MAIT TCR) recognizes MR1 presenting vitamin $B$ metabolites. Here we describe the structures of a human MAIT TCR in complex with human MR1 presenting a non-stimulatory ligand derived from folic acid and an agonist ligand derived from a riboflavin metabolite. For both vitamin B antigens, the MAIT TCR docks in a conserved manner above MR1, thus acting as an innate-like pattern recognition receptor. The invariant MAIT TCR $\alpha$-chain usage is attributable to MR1-mediated interactions that prise open the MR1 cleft to allow contact with the vitamin B metabolite. Although the non-stimulatory antigen does not contact the MAIT TCR, the stimulatory antigen does. This results in a higher affinity of the MAIT TCR for a stimulatory antigen in comparison with a non-stimulatory antigen. We formally demonstrate a structural basis for MAIT TCR recognition of vitamin B metabolites, while illuminating how TCRs recognize microbial metabolic signatures.

\footnotetext{
${ }^{1}$ Department of Biochemistry and Molecular Biology, School of Biomedical Sciences, Monash University, Clayton, Victoria 3800, Australia. ${ }^{2}$ Department of Microbiology \& Immunology, Peter Doherty Institute for Infection and Immunity, University of Melbourne, Parkville, Victoria 3010, Australia. ${ }^{3}$ Australian Research Council Centre of Excellence in Structural and Functional Microbial Genomics, Monash University, Clayton, Victoria 3800, Australia. ${ }^{4}$ Division of Chemistry \& Structural Biology, Institute for Molecular Bioscience, The University of Queensland, Brisbane, Queensland 4072, Australia. ${ }^{5}$ Queensland Institute of Medical Research and Australian Centre for Vaccine Development, Brisbane, Queensland 4006, Australia. ${ }^{6}$ Institute of Infection and Immunity, Cardiff University, School of Medicine, Heath Park, Cardiff CF14 4XN, UK. * These authors contributed equally to this work. Correspondence and requests for materials should be addressed to J.McC. (email: jamesm1@unimelb.edu.au) or to J.R. (email: jamie.rossjohn@monash.edu).
} 
$\mathrm{T}$ he $\alpha \beta$ T-cell receptor (TCR) binds peptide and lipid-based antigens presented by the major histocompatibility complex (MHC) and CD1 families, respectively. In addition, some TCRs can recognize small organic compounds presented by MHC-like molecules, although the basis of this interaction is unknown. Mucosal-associated invariant T (MAIT) cells are an abundant population of innate-like $\mathrm{T}$ cells in humans that reside in the peripheral blood, but are predominantly found in the gastrointestinal mucosa and liver ${ }^{1,2}$. Although the physiological role of MAIT cells is emerging, it is established that numerous bacteria and yeast activate MAIT cells ${ }^{3-6}$. MAIT cells also require the gut microbiota for their development, and are implicated in protective immunity and several disorders caused by aberrant immunity $y^{3,4,7-12}$. MAIT cells, like type I natural killer T-cells (NKT cells), rapidly secrete a range of cytokines upon activation through a receptor selected from the adaptive $\alpha \beta$ TCR repertoire that is expressed on their cell surface ${ }^{5}$. The NKT TCR specifically recognizes a variety of synthetic, self and foreign lipid-based ligands bound to CD1d $\mathrm{d}^{13,14}$, whereas the MAIT TCR is restricted to the ubiquitously expressed MHC-I-related molecule ${ }^{15}$, MR1, which presents vitamin B-based metabolites to MAIT cells ${ }^{16}$. Consistent with their innate-like phenotype, MAIT and NKT cells express a very restricted $\mathrm{T}$-cell repertoire, in contrast to the enormously diverse T-cell repertoire often observed in MHCrestricted immunity ${ }^{17-19}$. Human MAIT cells are characterized by an invariant TCR $\alpha$-chain (TRAV1-2-TRAJ33) paired with a limited array of TCR $\beta$-chains (TRBV6 or TRBV20) ${ }^{17}$, whereas human type I NKT cells express an invariant $\alpha$-chain (TRAV10TRAJ18) coupled to a TRBV25-1-encoded TCR $\beta$-chain ${ }^{20}$. A high level of conservation of MR1 in mammals, and the restricted MAIT TCR usage, strongly indicates an important and evolutionarily conserved function for the MAIT TCR-MR1 axis in immunity ${ }^{21}$. We recently described a family of vitamin B-related ligands presented by MR1 that exhibit variable potency for MAIT cells ${ }^{16}$. Central to understanding the MAIT cell function is the basis for the highly restricted MAIT TCR $\alpha$-chain selection, the nature of the MAIT TCR-MR1-Ag interaction, the mechanisms by which the MAIT TCR discriminates between MR1-bound vitamin B metabolites, and the basis of MAIT cell agonism.

Here we present the crystal structures of the MAIT TCR bound to MR1 presenting a non-agonist and an agonist antigen. We provide insight into the invariant MAIT TCR $\alpha$-chain usage, MR1 restriction and, importantly, provide definitive insight into TCR recognition of vitamin $B$ metabolites, thereby providing the molecular basis for MAIT cell agonism.

\section{Results}

Overview of the MAIT TCR-MR1-Ag complex. Although a folic acid metabolite (6-formyl pterin, 6-FP) did not activate MAIT cells, riboflavin derivatives, including 7-hydroxy-6-methyl8-D-ribityllumazine (RL-6-Me-7-OH), specifically stimulated MAIT cells in an MR1-restricted manner ${ }^{16}$. Although the MR1restricted ligands, including 6,7-dimethyl-8-D-ribityllumazine and reduced 6-hydroxymethyl-8-D-ribityllumazine, refolded very inefficiently with MR1 (thereby hampering structural studies), 6-FP and RL-6-Me-7-OH refolded sufficiently well with MR1 to enable structural studies to readily proceed. Accordingly, to definitively establish how a MAIT TCR binds stimulatory and non-stimulatory MR1-antigen complexes, we expressed and refolded a MAIT TCR (TRAV1-2-TRAJ33TRBV6-1) (not shown) and crystallized it in complex with MR1-6-FP and MR1-RL-6-Me-7-OH. Both MAIT TCR-MR1Ag ternary complexes were solved to a very high resolution (Table 1), and the electron density for the vitamin B metabolites
Table 1 | Data collection and refinement statistics.

\begin{tabular}{|c|c|c|}
\hline & $\begin{array}{l}\text { MAIT TCR- } \\
\text { MR1-6-FP }\end{array}$ & $\begin{array}{c}\text { MAIT TCR-MR1- } \\
\text { RL-6-Me-7-OH }\end{array}$ \\
\hline \multicolumn{3}{|l|}{ Data collection } \\
\hline Temperature & $100 \mathrm{~K}$ & $100 \mathrm{~K}$ \\
\hline Space group & C2 & $\mathrm{C} 2$ \\
\hline \multicolumn{3}{|l|}{ Cell dimensions } \\
\hline$a, b, c(\AA)$ & $217.23,69.56,142.89$ & $\begin{array}{c}218.37,71.20 \\
144.64\end{array}$ \\
\hline$\alpha, \beta, \gamma\left({ }^{\circ}\right)$ & $90,104.42,90$ & $90,104.86,90$ \\
\hline \multirow[t]{2}{*}{ Resolution $(\AA)$} & $50.00-2.00$ & 75.44-1.90 \\
\hline & $(2.11-2.00)$ & $(2.00-1.90)$ \\
\hline$R_{\text {p.i.m. }}{ }^{\star}$ & $5.9(36.6)$ & $7.5(49.8)$ \\
\hline$I / \sigma_{1}$ & $10.5(2.1)$ & $9.8(2.2)$ \\
\hline Completeness (\%) & $99.9(99.9)$ & $99.8(100.0)$ \\
\hline Total no. of. observations & 709,849 & $1,198,766$ \\
\hline No. of. unique observations & 139,624 & $1,68,903$ \\
\hline Multiplicity & $5.1(5.1)$ & $7.1(7.1)$ \\
\hline \multicolumn{3}{|l|}{ Refinement statistics } \\
\hline$R_{\text {factor }}^{\dagger}(\%)$ & 18.4 & 17.9 \\
\hline$R_{\text {free }}(\%)$ & 22.4 & 20.9 \\
\hline \multicolumn{3}{|l|}{ No. of atoms } \\
\hline Protein & 12,719 & 12,572 \\
\hline Ligand & 26 & 46 \\
\hline Water & 1,449 & 1,103 \\
\hline \multicolumn{3}{|l|}{ Ramachandran plot (\%) } \\
\hline Most favoured & 91.5 & 91.8 \\
\hline Allowed region & 8.5 & 8.2 \\
\hline \multicolumn{3}{|l|}{ B-factors $\left(\AA^{\dagger}\right)$} \\
\hline Protein & 30.0 & 29.5 \\
\hline Ligand & 22.2 & 21.6 \\
\hline r.m.s.d. bonds $(\AA)$ & 0.010 & 0.010 \\
\hline r.m.s.d. angles $\left({ }^{\circ}\right)$ & 1.05 & 1.03 \\
\hline \multicolumn{3}{|c|}{$\begin{array}{l}\text { Values in parentheses refer to the highest-resolution bin. } \\
{ }^{\star} R_{\text {pi.m. }}=\Sigma_{h k l}[1 /(N-1)]^{1 / 2} \Sigma_{i}\left|I_{h k l, i}-<I_{h k l}>\right| / \Sigma_{h k l}<I_{h k l}> \\
\dagger R_{\text {factor }}=\left(\Sigma|| \mathrm{F}_{\mathrm{o}}|-| \mathrm{F}_{\mathrm{c}}||\right) /\left(\Sigma\left|\mathrm{F}_{\mathrm{o}}\right|\right)-\text { for all data except as indicated in the next footnote. } \\
\ddagger 5 \% \text { of data were used for the } R_{\text {free }} \text { calculation. }\end{array}$} \\
\hline
\end{tabular}

within the MR1 clefts and that at the MAIT TCR-MR1-Ag interfaces were unambiguous (Supplementary Fig. S1). Apart from ligand-specific interactions (discussed below), the two MAIT TCR-MR1-Ag complexes are virtually identical. To provide a context for how TCRs can recognize vitamin B metabolites, peptides and lipids, we compared the MAIT TCRMR1-RL-6-Me-7-OH complex with a representative TCRMHC-I-peptide and a type I NKT TCR-CD1d- $\alpha$-galactosylceramide $\left(\alpha\right.$-GalCer) complex (Fig. 1a) ${ }^{22,23}$.

The MAIT TCR docked approximately centrally and orthogonally to the main axis of the MR1-Ag-binding cleft, interacting with residues spanning $61-72$ and 148-164 of the $\alpha 1$ and $\alpha 2$ helices of MR1, respectively (Fig. 1a,b). The MAIT TCR-MR1$\mathrm{Ag}$ docking topology starkly contrasted with the consensus type I NKT TCR-CD1d-lipid interaction, whereupon the NKT TCR docked parallel to and above the $\mathrm{F}^{\prime}$-pocket of the CD1d-antigen complex (Fig. 1a-c) ${ }^{13}$. Although TCRs can engage the MHC peptide in a range of docking modes ${ }^{24}$, the MAIT TCR-MR1-Ag complex was analogous to typical TCR-MHC-I-peptide docking, in which the $\alpha$ and $\beta$ chains of the MAIT TCR were positioned over the $\alpha 2$ and $\alpha 1$ helices of MR1, respectively (Fig. 1a,b). Indeed, there was similarity in the overall docking mode between a TRAV1-2 TCR-HLA-B ${ }^{*} 35: 01$-peptide complex ${ }^{22}$ and the MAIT TCR-MR1-RL-6-Me-7-OH complex. This similarity was surprising, given that the VB8.2 ${ }^{+}$TCRs adopt markedly different footprints on MHC and $\mathrm{CD} 1 \mathrm{~d}^{25}$. Further, the respective interatomic contacts differed markedly, which is partly due to a 
a

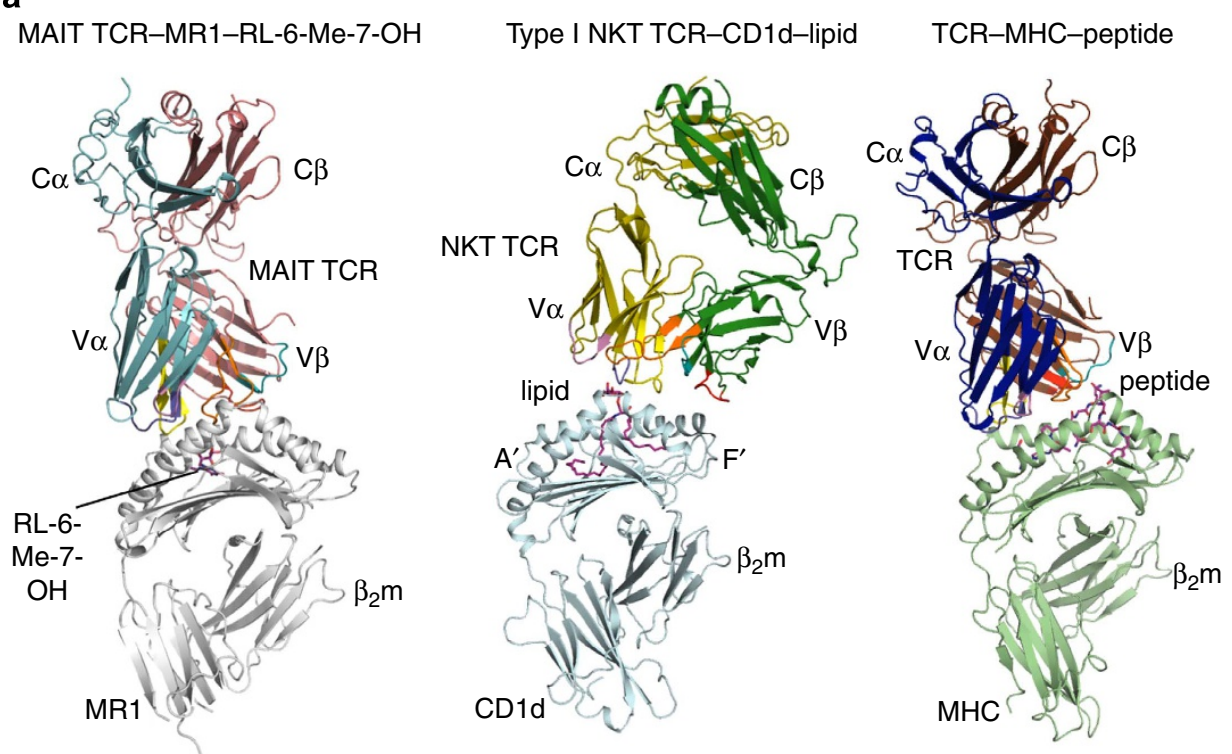

b
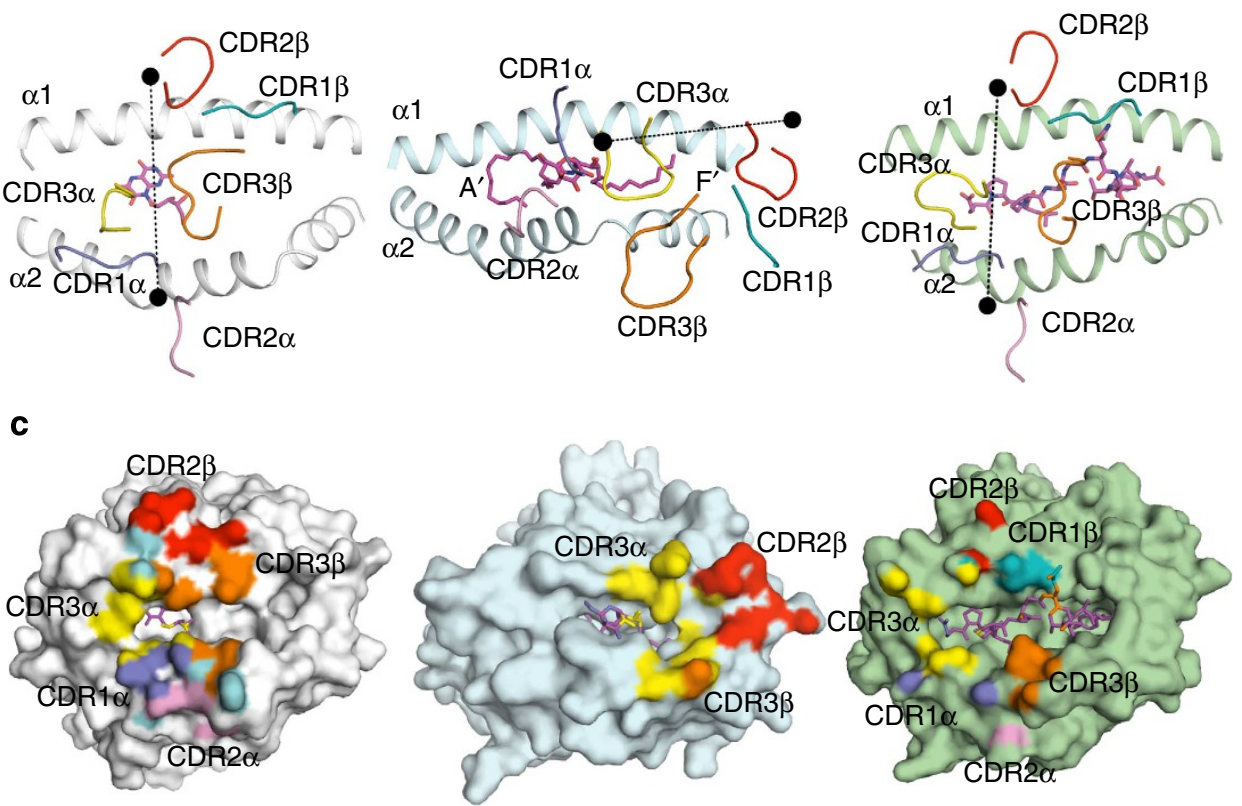

Figure 1 | Docking mode of the MAIT TCR in comparison with an NKT TCR that binds CD1d-lipid and a conventional TCR that binds MHC-I-peptide. (a) Left, MAIT TCR in complex with MR1-RL-6-Me-7-OH. MAIT TCR $\alpha$-chain, cyan; MAIT TCR $\beta$-chain, salmon; CDR1 $\alpha$, purple; CDR2 $\alpha$, pink, CDR3 $\alpha$, yellow; CDR1 $\beta$, teal; CDR2 $\beta$, red; CDR3 $\beta$, orange; RL-6-Me-7-OH, magenta; and MR1, grey. Middle, type I NKT TCR in complex with CD1d-lipid. NKT TCR $\alpha$-chain, yellow; NKT TCR $\beta$-chain, green; lipid, magenta; CD1d, pale cyan; CDR loops colour coding as in a. Right, conventional TRAV1-2 TCR in complex with an MHC-peptide TCR $\alpha$-chain, blue; TCR $\beta$-chain, brown; MHC, pale green; peptide, magenta; CDR loops colour coding as in a. (b) Left, MAIT TCRMR1-RL-6-Me-7-OH complex; middle, NKT TCR-CD1d-lipid complex; and right, conventional TRAV1-2 TCR-MHC-peptide complex viewed down into the antigen-binding cleft. Black-filled circles indicate the centre of mass for the $V \alpha$ and $V \beta$ domains; CDR loops colour coding as in $\mathbf{a}$. (c) Left, footprint of the MAIT TCR on the surface of MR1-RL-6-Me-7-OH; middle, footprint of the NKT TCR on the surface of CD1d-lipid; and right, footprint of the TRAV1-2 TCR on the surface of MHC-peptide. CDR loops colour coding as in $\mathbf{a}$.

lack of sequence identity between MR1 and MHC-I at the site of the MAIT TCR footprint (Fig. 1c, Supplementary Fig. S2). Moreover, there was little sequence identity between MR1 and the CD1 family at this interaction site, suggesting that there will be little in common between how TCRs can engage CD1 and MR1 (Supplementary Fig. S2). The buried surface area (BSA) at the MAIT TCR-MR1-Ag interface was approximately $1,200 \AA^{2}$, which falls within the upper end of the range of BSA values for TCR-MHC-I-peptide interactions (600-1,200 $\AA^{2}$ ) and outside the range of NKT TCR-CD1d-lipid interactions (760$\left.910 \AA^{2}\right)^{13,24,26}$. However, RL-6-Me-7-OH contributed merely $0.6 \%$ of the BSA at the MAIT TCR-MR1-RL-6-Me-7-OH interface, whereas the 6-FP ligand did not contribute at all. This low \% BSA contribution from the MAIT agonist ligand contrasts with the range that peptides contribute to the TCRMHC-I-peptide interface (10-25\% BSA), and is more analogous to how only a small component of lipid Ags are directly available for NKT TCR contact ${ }^{13,24}$. Thus, MR1 dominates the interactions 
with the MAIT TCR (Supplementary Tables S1 and S2), which was characterized mostly by hydrophobic contacts.

Basis of MAIT TCR $\beta$-gene usage. The $\alpha$ - and $\beta$-chain of the MAIT TCR contributed almost equally to the BSA at the MAIT TCR-MR1-Ag interface. The MAIT TCR $\beta$-chain is biased towards TRBV6-1, TRBV6-4 and TRBV20 usage ${ }^{17}$. Although the CDR1 $\beta$ loop did not contact MR1-Ag, the CDR2 $\beta$ loop abuts the $\alpha 1$ helix, and makes $13 \%$ BSA upon complexation (Fig. 1c). There are a large number of polar residues at the CDR2 $\beta-M R 1$ contact
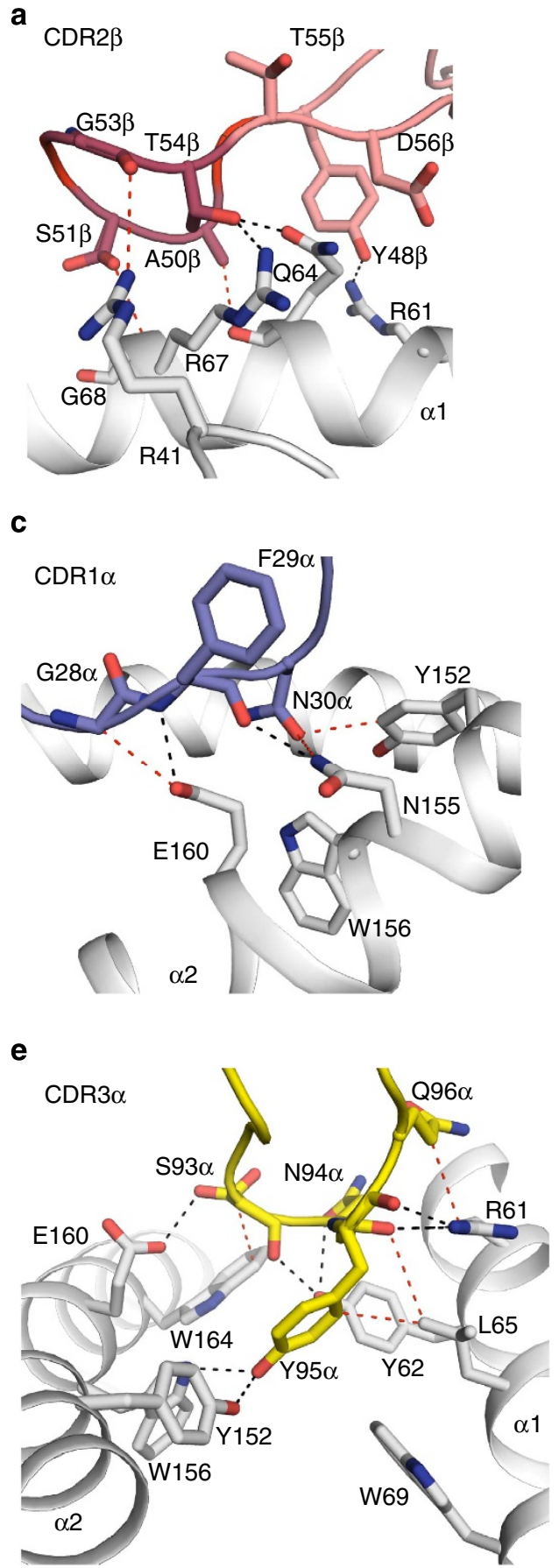

zone, but only one CDR2 $\beta$ residue (Thr54 $\beta$ ) H-bonds to MR1, and thus van der Waals contacts underpin the CDR2 $\beta-M R 1$ interaction (Fig. 2a). Nevertheless, three neighbouring framework residues (Tyr48 $\beta$, Thr55 $\beta$ and Asp56 $\beta$ ) that flank the CDR2 $\beta$ loop also add to the TCR $\beta$-chain-MR1 interface (8.4\% BSA) (Fig. 1c), which includes a H-bond between Tyr48 $\beta^{\mathrm{OH}}$ and Arg61 from MR1 (Fig. 2a). Mostly, the V $\beta$-mediated interactions seem non-ideally disposed to interact with MR1, consistent with previous mutagenesis studies on the TRBV20-MAIT TCR, which suggested that no individual V $\beta$ residue is essential for MAIT cell activation $^{27}$. Further, there is no sequence identity in the
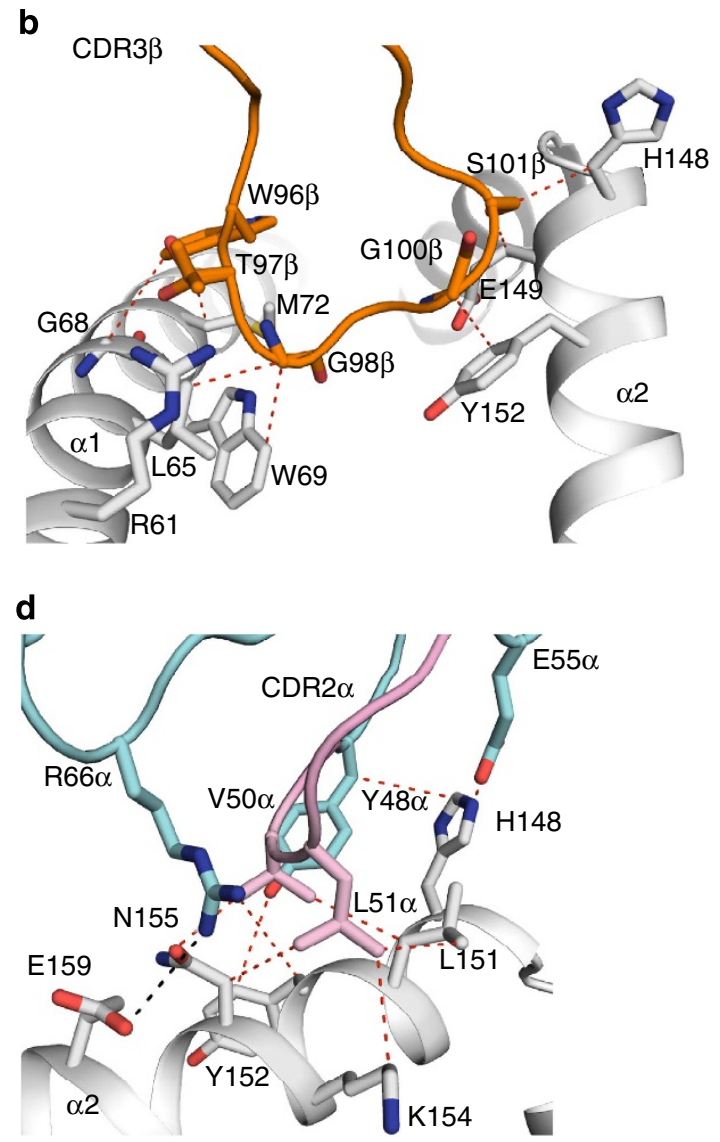

Figure 2 | Contacts between MAIT TCR and MR1-RL-6-Me-7-OH. (a) Contacts between CDR2 $\beta$ and CDR2 $\beta$ framework residues and MR1. (b) Contacts between CDR3 $\beta$ and MR1. (c) Contacts between CDR1 $\alpha$ and MR1. (d) Contacts between CDR2 $\alpha$ and TCR $\alpha$ chain framework residues and MR1. (e) Contacts between CDR3 $\alpha$ and MR1. MR1, grey; CDR2 $\beta$, red; CDR2 $\beta$ framework, salmon; CDR3 $\beta$, orange; CDR1 $\alpha$, purple; CDR2 $\alpha$, pink; TCR $\alpha$ chain framework, cyan; and CDR3 $\alpha$, yellow. $\mathrm{H}$-bond and van der Waals interactions are shown in black- and red-dashed lines, respectively. 
corresponding CDR1 $\beta$ and $2 \beta$ loops between the TRBV6-1/6-4 and TRBV20 genes ${ }^{27}$. This suggests that the V $\beta$ bias in the MAIT TCR usage may be attributable to preferential pairing with the MAIT TCR $\alpha$-chain to generate MR1-restricted TCRs.

Surprisingly, the majority of the TCR $\beta$-chain interactions $(24.6 \% \mathrm{BSA})$ arose from the non-germline-encoded CDR3 $\beta$ loop, which, although proximal to the bound Ag, exclusively contacted MR1 directly (Fig. 1c). The CDR3 $\beta-M R 1$ interactions were chiefly hydrophobic in nature (Supplementary Tables S1 and S2). Although interactions via Gly100 $\beta$ and Ser $101 \beta$ contributed to the CDR3 $\beta-M R 1$ interface, a ${ }^{96} \mathrm{Trp}-\mathrm{Thr}-\mathrm{Gly}{ }^{98}$ ' sequence had a dominant role in the CDR3 $\beta$-mediated contacts. Trp96 $\beta$ lies flat against the $\alpha 1$ helix of MR1, packing against Gly68 and Met72, whereas Thr97 $\beta$ and Gly98 $\beta$ form hydrophobic interactions with Arg61, Leu65 and Trp69, with Leu65 having been previously implicated as an important contact residue (Fig. 2b) ${ }^{27}$. The diverse nature of the CDR3 $\beta$ loop in the MAIT TCR repertoire ${ }^{17}$, and its location within the MAIT TCR-MR1-Ag complex, indicates that $\mathrm{CDR} 3 \beta$ variability may have a direct impact on the extent of self-reactivity with MR1-antigen.

Basis of MAIT TCR invariant gene usage. MAIT cells are characterized by germline-encoded invariant TCR $\alpha$-chain usage (TRAV1-2-TRAJ33), with a variable residue encoded at the V-J junction, which does not contact MR1-Ag (not shown). The $\mathrm{CDR} 1 \alpha, \mathrm{CDR} 2 \alpha, \mathrm{V} \alpha$ framework region and the CDR $3 \alpha$ loops contributed $6.8,11.0,12.0$ and $22 \%$ to the BSA of the MAIT TCR-MR1-Ag interface, respectively (Fig. 1c). Accordingly, the CDR3 $\alpha$ loop of the MAIT TCR $\alpha$-chain made the greatest contribution to the interface, consistent with the selection of the invariant TRAJ33 gene segment in MAIT cells (Fig. 1c). The

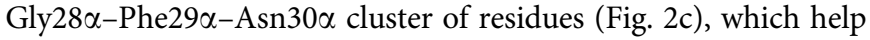
fix the CDR1 $\alpha$ loop to MR1, are encoded exclusively by the
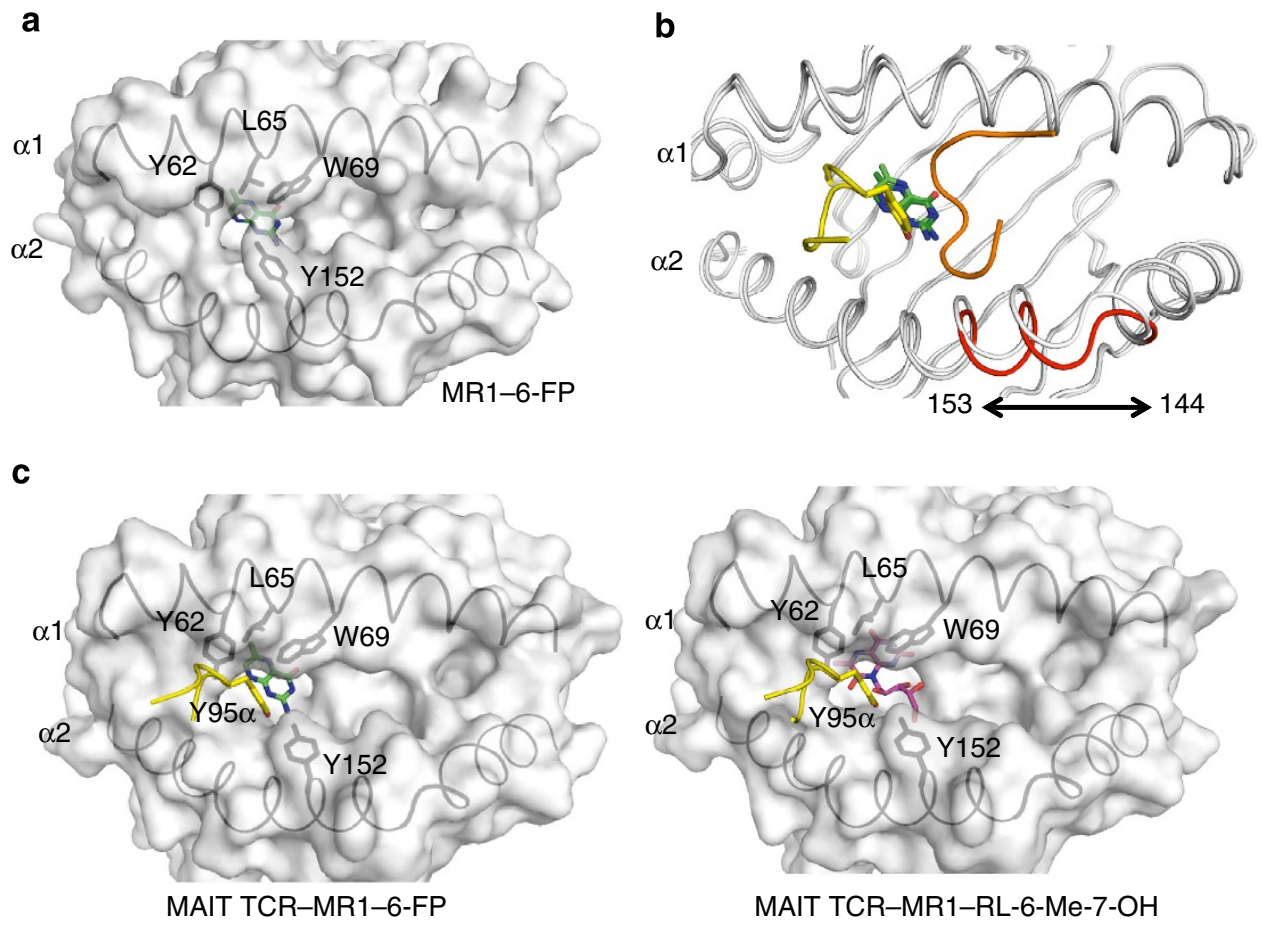

TRAV1 gene family. Moreover, Phe29 $\alpha$ is totally conserved across all mammalian TRAV1-2 orthologues (Supplementary Fig. S3), highlighting the functional role of this residue in MAIT-MR1 coevolution. The CDR2 $\alpha$ loop resides at the periphery of the interface (Fig. 1b), with two 'hydrophobic pegs' (Val50 $\alpha$ and Leu51 $\alpha$ ) sitting in an MR1 'notch' that is lined by Leu151 and Tyr152, and the aliphatic moieties of Lys154 and Asn155 (Fig. 2d). Interestingly, the Leu151Ala mutant can moderately increase MAIT TCR autoreactivity ${ }^{21,27}$, thereby further highlighting the role of this MR1 residue in MAIT TCR recognition. Flanking the 'hydrophobic peg' are two $V \alpha$-encoded framework residues, Tyr $48 \alpha$ and Arg66 $\alpha$, which collectively form a series of interactions with MR1 (Fig. 2d). Tyr48 $\alpha$ packs against His148 and points towards Tyr152, whereas Arg66 $\alpha$ salt bridges to Glu159 and H-bonds with Asn155 (Fig. 2d). Evolutionarily, these CDR2 $\alpha$-framework positions are either totally conserved across mammalian TRAV1-2 orthologues or exhibit synonymous substitutions (Supplementary Fig. S3).

The TRAJ33-encoded CDR3 $\alpha$ loop sits between the helical jaws of the MR1 cleft, having a principal role in contacting both the $\alpha 1$ and $\alpha 2$ helices of MR1 and the antigen itself (discussed below) (Supplementary Tables S1 and S2). Three residues at the tip of the CDR3 $\alpha$ loop (Ser93 $\alpha$, Asn94 $\alpha$, Tyr95 $\alpha$ ) engage MR1 and this particular germline configuration is not present in any of the other 60 TRAJ genes (Fig. 2e). Three TRAJ33-encoded residues have a prominent role in the interaction: Ser93 $\alpha$ H-bonds to Glu160, and its backbone H-bonds to Tyr62 ${ }^{\mathrm{OH}}$; Asn $94 \alpha$ backbone packs against and H-bonds to Arg61. Tyr95 $\alpha$ acts as a lynch-pin, in that it protrudes deep into the MR1-Ag-binding cleft, with its hydroxyl group $\mathrm{H}$-bonding to $\mathrm{Tyr} 152^{\mathrm{OH}}$, while its aromatic ring is surrounded by a nest of aromatic residues that includes Tyr62, Trp69, Tyr152 and Trp156 (Fig. 2e). Accordingly, Tyr $95 \alpha$ represents a principal player in the invariant TRAJ33 use of the MAIT TCR. Of note, Tyr95 $\alpha$ is totally conserved across all

Figure 3 | Conformational flexibility of the MR1-antigen-binding cleft. (a) Antigen-binding cleft of MR1 with 6-FP bound. The surface is transparent to show 6-FP. (b) Cartoon overlay of the antigen-binding clefts of MR1-6-FP and MAIT TCR-MR1-6-FP. Movement of the MR1 $\alpha 2$ helix region 144-153 upon MAIT TCR binding is shown in red. (c) Left, antigen-binding cleft of MR1 within the MAIT TCR-MR1-6-FP complex. Right, antigen-binding cleft of MR1 within the MAIT TCR-MR1-RL-6-Me-7-OH complex. The surface is transparent. MR1, grey; 6-FP in MR1-6-FP, dark green; 6-FP in MAIT TCR-MR1-6-FP complex, green; RL-6-Me-7-OH, magenta; CDR3 $\alpha$, yellow. 
known mammalian TRAJ33 orthologues. Collectively, only TRAV1-2-TRAJ33 and none of the other 102 variable or joining genes in the human TCR $\alpha$-locus encode the correct architecture required to maintain archetypal docking to MR1. In addition, key anchor sites within these receptor genes (Asn $30 \alpha$, Tyr $48 \alpha$, Val50 $\alpha$, Leu51 $\alpha$ and Arg66 $\alpha$, Ser93 $\alpha$, Asn $94 \alpha, \operatorname{Tyr} 95 \alpha$ and Gln96 $)$ are highly conserved across eutherian and marsupial mammal orthologues, which suggest that the mode of MAIT TCR-MR1 recognition has remained relatively unchanged for $150-180$ million years ${ }^{28}$.

MR1 plasticity upon MAIT TCR binding. In addition to determining the nature of the TCR contacts with the vitamin B metabolites, a comparison of the non-liganded TRAV1-2TRAJ33-TRBV20 MAIT TCR structure and the binary MR16-FP complex ${ }^{16,27}$ allowed us to simultaneously address the extent of conformational change within the MAIT TCR $\alpha$-chain and MR1-6-FP upon ligation. Within the binary MR1-6-FP complex, the 6-FP moiety was sequestered closely within the MR1 cleft, such that 6-FP was virtually inaccessible for direct MAIT TCR contact (Fig. 3a) ${ }^{16}$. Interestingly, while the MAIT TCR $\alpha$-chain and the 6-FP ligand itself did not move upon MAIT TCR-MR16-FP ligation, the MR1-Ag-binding cleft was more malleable (Fig. 3b). To accommodate the incoming Tyr95 $\alpha$ residue from the CDR3 $\alpha$ loop and the neighbouring CDR3 $\beta$ loop, a region of the $\alpha 2$-helix (144-153) of MR1 was 'prised open' upon MAIT TCR engagement (Fig. 3b). This prising apart of the MR1 cleft, and the displacement of Tyr152, resulted in greater solvent exposure of 6FP (Fig. 3c). Nevertheless, there was no direct contact between the MAIT TCR and 6-FP in the ternary complex, although Tyr $95 \alpha \mathrm{OH}$ formed a water-mediated $\mathrm{H}$-bond with the $\mathrm{N} 1$ atom of 6-FP (Fig. 4a). Notably, the lack of direct MAIT TCR-6-FP contacts was consistent with 6-FP being a non-stimulatory antigen and the very low affinity of this interaction $\left(K_{\mathrm{D}(\mathrm{eq})}>300 \mu \mathrm{M}\right)$. Of note, 6-FP is a competitive inhibitor of MAIT activation as it reduces RL-6-Me-7-OH and rRL-6- $\mathrm{CH}_{2} \mathrm{OH}$-induced activation of Jurkat cells transduced with three distinct MAIT TCRs in a dosedependent manner (Fig. 5).

Interaction with vitamin B metabolites. The overall features of the MAIT TCR-MR1-6FP and MAIT TCR-MR1-RL-6-Me-7$\mathrm{OH}$ complexes were very similar to each other, including the more open MR1-Ag-binding cleft that increased the solvent accessibility of RL-6-Me-7-OH (Fig. 3c, right panel). However, key differences between the complexes included the MR1-6-FP contacts differing from those of MR1-RL-6-Me-7-OH, and RL-6Me-7-OH directly contacting the MAIT TCR. First, while 6-FP formed a Schiff base with Lys43 (Fig. 4a), RL-6-Me-7-OH was non-covalently bound within MR1, which resulted in Lys43 drifting away from the ligand (Fig. 4b). Second, and surprisingly, the orientation of RL-6-Me-7-OH within the aromatic cradle of MR1 differed dramatically from that of 6-FP. Namely, RL-6-Me$7-\mathrm{OH}$ is rotated $\sim 75^{\circ}$ with respect to 6-FP, such that the MR1mediated contacts between the respective ring systems differ between 6-FP and RL-6-Me-7-OH (Fig. 4b). The tilted conformation causes the ribityl moiety of RL-6-Me-7-OH to lean towards, and form a series of H-bonds with, Arg9 and Arg94, two evolutionarily conserved residues found within MR1 (ref. 16). These two Arg residues act as a platform to present the ribityl moiety in a fixed conformation to enable a direct $\mathrm{H}$-bond with Tyr95 $\alpha^{\mathrm{OH}}$ from the CDR3 $\alpha$ loop of the MAIT TCR (Fig. 4c). Although the $\mathrm{Tyr} 95 \alpha^{\mathrm{OH}_{-}}$-mediated interaction represented the sole direct contact between the MAIT TCR and the stimulatory ligand, it nevertheless was associated with an increased affinity of interaction $\left(K_{\mathrm{D}(\mathrm{eq})}=1.65 \pm 0.16 \mu \mathrm{M}\right.$ Fig. $\left.6 \mathrm{a}\right)$. The importance

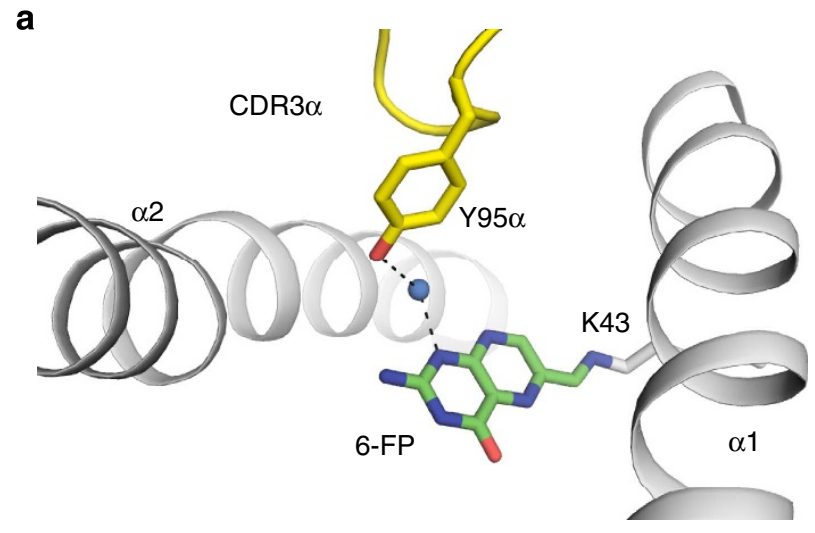

b

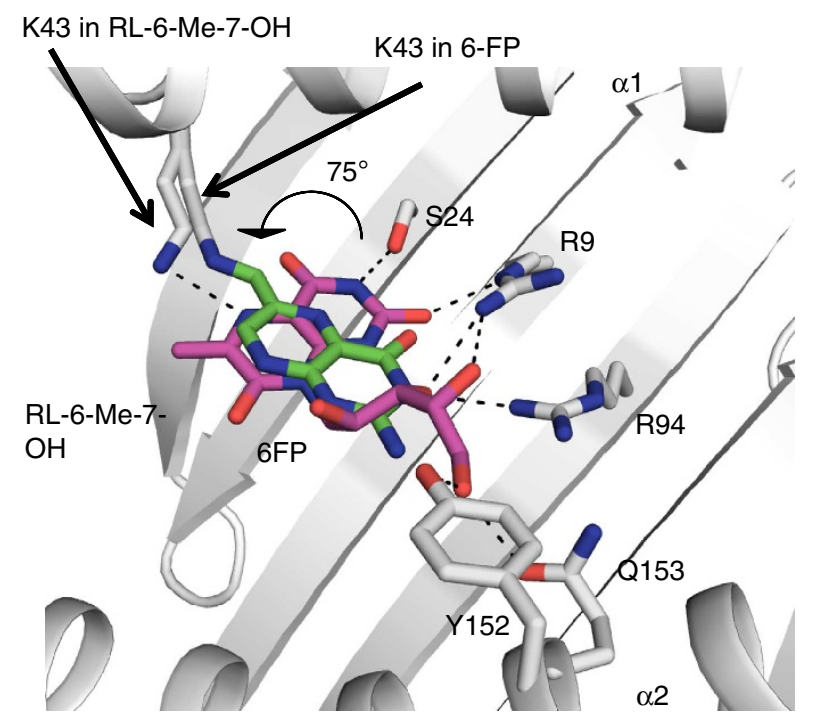

C

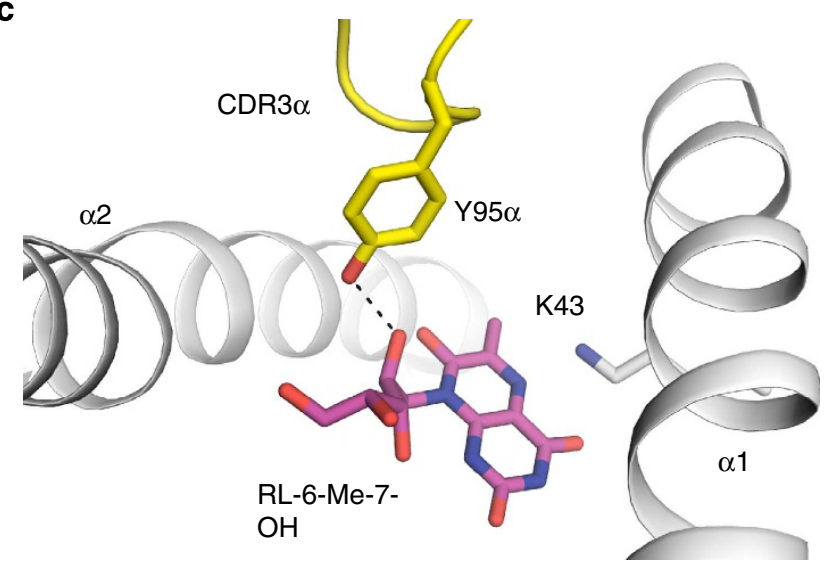

Figure 4 | Differences between MAIT TCR-MR1-6-FP and MAIT TCRMR1-RL-6-Me-7-OH complexes. (a) Water-mediated interaction between CDR3 $\alpha$ of MAIT TCR and 6-FP. (b) Overlay of MAIT TCR-MR1-6-FP and MAIT TCR-MR1-RL-6-Me-7-OH complexes. Arrow indicates $75^{\circ}$ rotation of RL-6-Me-7-OH compared with 6-FP. (c) H-bond interaction between CDR3 $\alpha$ of MAIT TCR and RL-6-Me-7-OH. MR1, grey; CDR3 $\alpha$, yellow; 6-FP, green; RL-6-Me-7-OH, magenta; blue circle, water molecule; $\mathrm{H}$-bond interactions are shown in black dashed lines.

of this Ag-centric interaction was evident from the impact of the Tyr $95 \alpha$ Phe mutation, which markedly reduced the affinity $\left(K_{\mathrm{D}(\mathrm{eq})}=33.89 \pm 2.22 \mu \mathrm{M}\right)$ towards the potent ligand 
a

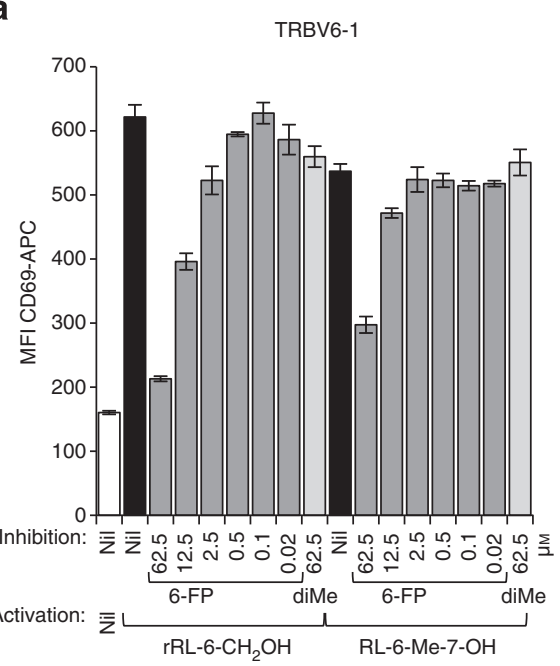

b

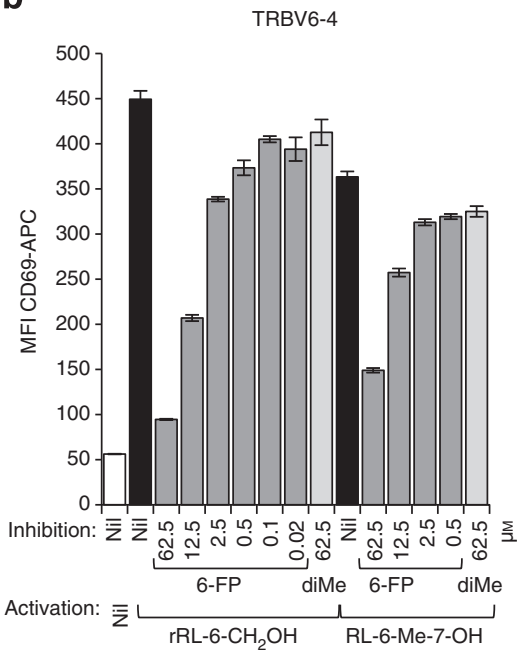

C

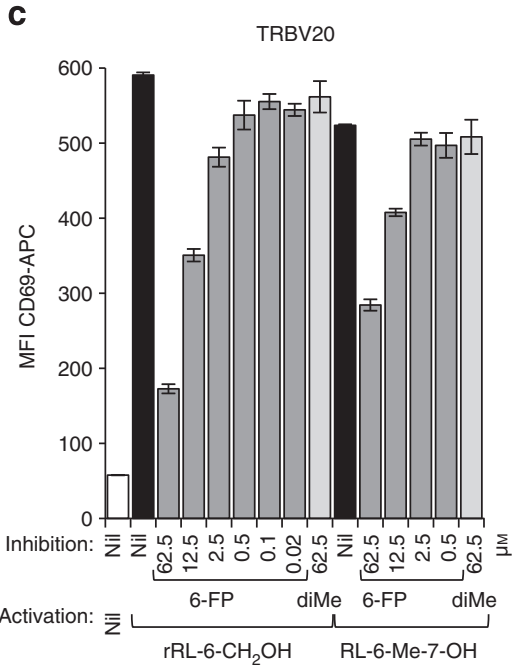

Figure 5 | MAIT TCR inhibition by 6-FP. C1R cells expressing human MR1 were incubated together with Jurkat.MAIT cells (10 5 each) expressing (a) TRBV6-1, (b) TRBV6-4 or (c) TRBV20 TCRs and 6-FP or a control, pterin 6,7-dimethylpterin (di-Me), for 30 min prior to addition of RL-6-Me-7-OH or rRL$6-\mathrm{CH}_{2} \mathrm{OH}$, and incubation overnight. Mean fluorescence intensity of CD69 expression for gated-Jurkat.MAIT cells. Mean \pm s.e.m. of triplicate samples.

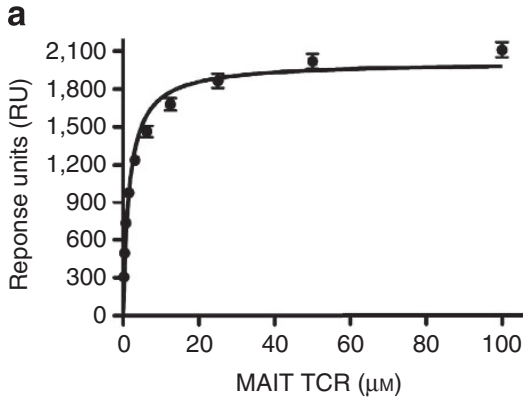

c b

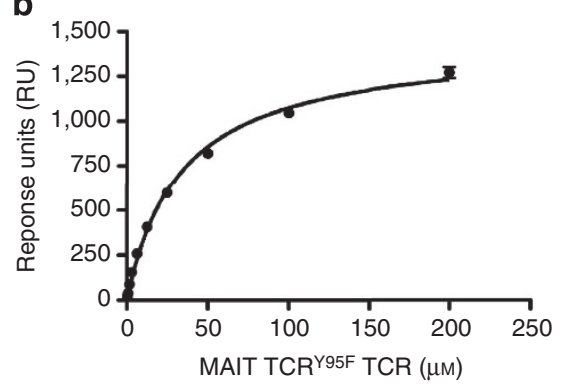

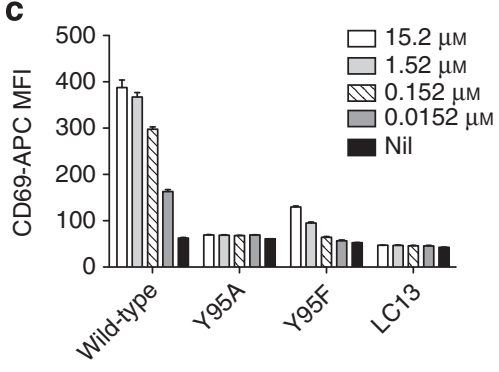

Figure 6 | MAIT TCR affinity for MR1 bound to antigens. SPR affinity-based measurements (at $25^{\circ} \mathrm{C}$ ) of the MAIT TCR binding to a potent agonist, rRL-6- $\mathrm{CH}_{2} \mathrm{OH}$. Saturation binding curves as determined by SPR for wild-type MAIT TCR (a) or MAIT TCR ${ }^{\mathrm{S} 95 \mathrm{~F}}$ TCR (b). Data are representative of two independent experiments with error bars representing s.e.m. of the duplicates. (c) Activation of SKW-MAIT-TRBV6-1 cells with rRL-6-CH ${ }_{2} \mathrm{OH}$. SKW cells transduced with genes encoding a MAIT TCR expressing wild-type or mutants Y95A, or Y95F invariant TCR $\alpha$-chain (TRAV1-2-TRAJ33) paired with a

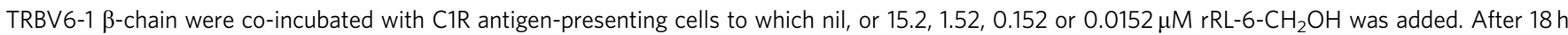
SKW cells were stained and analysed by flow cytometry for cell-surface expression of CD69. Shown is the mean fluorescence intensity of staining of CD69APC on the Y-axis. Control SKW cells expressing the EBV-specific $\alpha \beta$ TCR LC13 (ref. 40) were not activated by rRL-6-CH $\mathrm{CH}_{2} \mathrm{OH}$. Data are representative of two independent experiments with error bars representing the s.e.m. of triplicate samples.

rRL-6- $\mathrm{CH}_{2} \mathrm{OH}$, as judged by SPR and cell-based activation assays (Fig. 6b,c). Thus, the principles differentiating non-stimulatory antagonist ligands and stimulatory MAIT cell antigens can be attributed to a single $\mathrm{H}$-bond to the ligand itself.

\section{Discussion}

Understanding how the immune system of the human mucosa interacts with the microbial products of the commensal microbiota and invading pathogens represents a key emerging area. We describe the molecular basis of how the immune system recognizes microbial metabolic products. Although the basis for interactions between TCRs and MHC-peptide or CD1lipid complexes is established ${ }^{25}$, molecular insights into the newly discovered capacity of TCRs to interact with small organic molecules presented by MHC-like molecules is lacking. Our findings formally detail how a TCR recognizes vitamin B metabolites and, more broadly, TCR ligation to small organic metabolites bound by an Ag-presenting molecule. 
MAIT TCR-MR1-antigen recognition shares a mixture of key features associated with innate-like and adaptive TCR recognition $^{13,24}$. Namely, the overall MAIT TCR-MR1-Ag docking topology was more similar to that of typical TCR-peptide-MHC complexes and a Type II NKT TCR-CD1d-sulfatide complex ${ }^{29,30}$. Nevertheless, the germline-encoded region of the rigid MAIT TCR contacted the minimally exposed vitamin B antigen and remoulded the MR1-Ag-binding cleft upon ligation; these features are more typical of the innate-style interactions and indicate that the MAIT TCR is an innate-like pattern recognition receptor targeted towards vitamin $\mathrm{B}$ metabolites. For example, the invariant NKT TCR was shown to mould the CD1d-antigenbinding cleft and deform $\beta$-linked ligands upon complexation ${ }^{31,32}$. A molecular basis for the invariant $\alpha$-chain usage of the MAIT TCR is presented, showing that only a single residue within the TRAJ33 region contacts the vitamin-B-derived antigen. Thus, the principles differentiating non-stimulatory ligands and stimulatory MAIT cell antigens were attributable to a single $\mathrm{H}$-bond to the ligand itself. Accordingly, understanding the molecular basis of human MAIT TCR-MR1-antigen recognition and the general principles underscoring MAIT cell agonism provides key insight into how an evolutionarily conserved and abundant innate-like T-cell population can sense microbe-derived vitamin B metabolites.

\begin{abstract}
Methods
MR1 and TCR preparation. Genes encoding soluble human MR1, $\beta 2 \mathrm{~m}$, TRAV12-TRAJ33 and TRBV6-1 were expressed for $4 \mathrm{~h}$ in BL21 Escherichia coli after induction with $1 \mathrm{~mm}$ isopropyl $\beta$-D-1-thiogalactopyranoside. E. coli were pelleted and resuspended in a buffer containing $50 \mathrm{mM}$ Tris, $25 \%$ (w/v) sucrose, $1 \mathrm{mM}$ EDTA and $10 \mathrm{mM}$ DTT $\mathrm{pH}$ 8.0. Inclusion-body protein was then extracted by lysis of bacteria in a buffer containing $50 \mathrm{mM}$ Tris $\mathrm{pH} 8.0,1 \%(\mathrm{w} / \mathrm{v})$ Triton X-100, $1 \%\left(\mathrm{w} / \mathrm{v}\right.$ ) sodium deoxycholate, $100 \mathrm{mM} \mathrm{NaCl}, 10 \mathrm{mM}$ DTT, $5 \mathrm{mM} \mathrm{MgCl}_{2}$ and $1 \mathrm{mg}$ DNaseI per litre of starting culture; the subsequent steps involved homogenization with a polytron homogenizer, centrifugation and washing inclusionbody protein sequentially, with (1) a buffer containing $50 \mathrm{mM}$ Tris $\mathrm{pH} 8.0,0.5 \%$ Triton X-100, $100 \mathrm{mM} \mathrm{NaCl}, 1 \mathrm{mM}$ EDTA and $1 \mathrm{mM}$ DTT, and (2) a buffer containing $50 \mathrm{mM}$ Tris pH 8.0, $1 \mathrm{mM}$ EDTA and $1 \mathrm{mM}$ DTT. Inclusion-body protein was then resuspended in a buffer containing $20 \mathrm{mM}$ Tris $\mathrm{pH} 8.0,8 \mathrm{M}$ urea, $0.5 \mathrm{mM}$ EDTA and $1 \mathrm{mM}$ DTT, and after centrifugation the supernatant containing solubilized, denatured inclusion-body protein was collected and stored at $-80^{\circ} \mathrm{C}$
\end{abstract}

Refolding of MR1 and MAIT TCR. The method for refolding and purifying the MR1- $\beta 2 \mathrm{~m}$-ligand complex is based on a similar methodology to that used for the classical MHC heavy-chain- $\beta 2 \mathrm{~m}$-peptide complex described in Kjer-Nielsen et al. ${ }^{16}$ and Garboczi et al. ${ }^{33}$ The refolded MR1- $\beta 2 \mathrm{~m}$-ligand complex was then purified by sequential DEAE (GE Healthcare) anion exchange, S75 16/60 (GE Healthcare) gel filtration and MonoQ (GE Healthcare) anion exchange chromatography. The TRAV1-2-TRAJ33-TRBV6-1 MAIT TCR was expressed, refolded and purified essentially as previously described ${ }^{27}$. Both the MR1- $\beta 2 \mathrm{~m}-$ ligand and MAIT TCR were concentrated to $6 \mathrm{mg} \mathrm{ml}^{-1}$ and mixed in a ratio of 1:1 prior to crystallization.

Structure determination. Crystals of MAIT TCR-MR1-6-FP and MAIT TCRMR1-RL-6-Me-7-OH complexes diffracted to $2 \AA$ or better and belong to the space group $C 2$, with two molecules within the asymmetric unit. The data were processed using Mosflm version 7.0.5 (ref. 34) and scaled using SCALA from the CCP4 Suite $^{35}$. The data for MAIT TCR-MR1-6-FP were solved by the molecular replacement method using PHASER in CCP4, with MR1 (PDB code 4GUP) without the ligand and ELS4 TCR (PDB code 2NX5) without the loop region as a search model. The data for MAIT TCR-MR1-RL-6-Me-7-OH complex were solved using MAIT TCR-MR1-6-FP, without the ligand as a model. To prevent model bias, the $R_{\text {free }}$ set of the MAIT TCR-MR1-6-FP data was used in the experimental intensities scaling using SCALA as well as the implementation of the simulated annealing protocol in Phenix ${ }^{36}$. RL-6-Me-7-OH was modelled using the Dundee PRODRG2 server ${ }^{37}$. Both structures were refined using BUSTER 2.10 (ref. 38). Model building was carried out using COOT. The quality of the structure was validated at the Research Collaboratory for Structural Bioinformatics Protein Data Bank Data Validation and Deposition Services. All molecular graphics representations were created using $\mathrm{PyMOL}^{39}$. Surface area calculations were carried out using Areaimol within the CCP4 suite ${ }^{35}$. See Table 1 for data collection and refinement statistics.
Surface plasmon resonance. All surface plasmon resonance (SPR) experiments were conducted at $25^{\circ} \mathrm{C}$ on a BIAcore 3000 instrument using HBS buffer $(10 \mathrm{mM}$ HEPES- $\mathrm{HCl}(\mathrm{pH} 7.4), 150 \mathrm{mM} \mathrm{NaCl}$ ). The biotinylated-MR1-Ag complexes were immobilized to an SA-Chip (GE) with a surface density of approximately 2,000 RU. Various concentrations of 6-1 MAIT TCR or MAIT TCR Y95F TCR (1.17$300 \mu \mathrm{M})$ were injected over the captured-MR1-Ag at $10 \mu \mathrm{min}^{-1}$. The final response was calculated by subtracting the response of the blank flow cell alone from the MAIT TCR-MR1-Ag complex. The equilibrium data were analysed using GraphPad Prism.

Assays of Jurkat cells transduced with MAIT TCR. Jurkat cells transduced with a MAIT TCR comprising the TRAV1-2-TRAJ33 invariant $\alpha$-chain and the TRBV6-1, TRBV6-4 or TRBV20 $\beta$-chains were tested for activation by coincubation with ribityl lumazine compounds and $\mathrm{C} 1 \mathrm{R}$ antigen-presenting cells expressing MR1. For inhibition experiments, 6-FP or a control pterin 6,7-dimethylpterin was added to C1R.MR1 cells $\left(10^{5}\right) 30 \mathrm{~min}$ before the addition of activating ribityl lumazine compounds RL-6-Me-7-OH and rRL-6- $\mathrm{CH}_{2} \mathrm{OH}$, after which Jurkat.MAIT cells $\left(10^{5}\right)$ were added for $16 \mathrm{~h}$. Cells were subsequently stained with PE-conjugated anti-CD3 and APC-conjugated anti-CD69 antibodies before analysis by flow cytometry. Activation of Jurkat.MAIT cells was measured by an increase in surface CD69 expression.

\section{References}

1. Treiner, E. et al. Selection of evolutionarily conserved mucosal-associated invariant T cells by MR1. Nature 422, 164-169 (2003).

2. Tang, X.-Z. et al. IL-7 licenses activation of human liver intrasinusoidal mucosal-associated invariant T cells. J. Immunol. 190, 3142-3152 (2013).

3. Le Bourhis, L. et al. Antimicrobial activity of mucosal-associated invariant T cells. Nat. Immunol. 11, 701-708 (2010).

4. Gold, M. C. et al. Human mucosal associated invariant T cells detect bacterially infected cells. PLoS Biol. 8, e1000407 (2010).

5. Godfrey, D. I., Rossjohn, J. \& McCluskey, J. Fighting infection with your MAITs. Nat. Immunol. 11, 693-695 (2010).

6. Gold, M. C. \& Lewinsohn, D. M. Co-dependents: MR1-restricted MAIT cells and their antimicrobial function. Nat. Rev. Microbiol. 11, 14-19 (2013).

7. Miyazaki, Y., Miyake, S., Chiba, A., Lantz, O. \& Yamamura, T. Mucosalassociated invariant $\mathrm{T}$ cells regulate $\mathrm{Th} 1$ response in multiple sclerosis. Int. Immunol. 23, 529-535 (2011).

8. Le Bourhis, L. et al. Mucosal-associated invariant T cells: unconventional development and function. Trends Immunol. 32, 212-218 (2011).

9. Chua, W. J. et al. Polyclonal mucosa-associated invariant T cells have unique innate functions in bacterial infection. Infect. Immun. 80, 3256-3267 (2012).

10. Cosgrove, C. et al. Early and non-reversible decrease of CD161 + + /MAIT cells in HIV infection. Blood 121, 951-961 (2012).

11. Leeansyah, E. et al. Activation, exhaustion and persistent decline of the antimicrobial MR1-restricted MAIT cell population in chronic HIV-1 infection. Blood 121, 1124-1135 (2012).

12. Vera, G. et al. Cernunnos deficiency reduces thymocyte life span and alters the T cell repertoire in mice and humans. Mol. Cell. Biol. 33, 701-711 (2013).

13. Rossjohn, J., Pellicci, D. G., Patel, O., Gapin, L. \& Godfrey, D.I. Recognition of CD1d-restricted antigens by natural killer T cells. Nat. Rev. Immunol. 12, 845-857 (2012).

14. Godfrey, D. I. \& Rossjohn, J. New ways to turn on NKT cells. J. Exp. Med. 208, 1121-1125 (2011).

15. Huang, S. et al. Evidence for MR1 antigen presentation to mucosal-associated invariant T cells. J. Biol. Chem. 280, 21183-21193 (2005).

16. Kjer-Nielsen, L. et al. MR1 presents microbial vitamin B metabolites to MAIT cells. Nature 491, 717-723 (2012).

17. Tilloy, F. et al. An invariant T Cell receptor alpha chain defines a novel TAPindependent major histocompatibility complex class Ib-restricted alpha/beta T Cell subpopulation in mammals. J. Exp. Med. 189, 1907-1921 (1999).

18. Turner, S. J., Doherty, P. C., McCluskey, J. \& Rossjohn, J. Structural determinants of T-cell receptor bias in immunity. Nat. Rev. Immunol. 6, 883-894 (2006).

19. Godfrey, D. I. et al. Antigen recognition by CD1d-restricted NKT T cell receptors. Semin. Immunol. 22, 61-67 (2010).

20. Bendelac, A., Savage, P. B. \& Teyton, L. The biology of NKT cells. Annu. Rev. Immunol. 25, 297-336 (2007).

21. Huang, S. et al. MR1 antigen presentation to mucosal-associated invariant T cells was highly conserved in evolution. Proc. Natl Acad. Sci. USA 106, 8290-8295 (2009).

22. Tynan, F. E. et al. A T cell receptor flattens a bulged antigenic peptide presented by a major histocompatibility complex class I molecule. Nat. Immunol. 8, 268-276 (2007).

23. Borg, N. A. et al. CD1d-lipid-antigen recognition by the semi-invariant NKT T-cell receptor. Nature 448, 44-49 (2007). 
24. Gras, S. et al. A structural voyage toward an understanding of the MHC-Irestricted immune response: lessons learned and much to be learned. Immunol. Rev. 250, 61-81 (2012).

25. Godfrey, D. I., Rossjohn, J. \& McCluskey, J. The fidelity, occasional promiscuity, and versatility of $\mathrm{T}$ cell receptor recognition. Immunity $\mathbf{2 8}$, 304-314 (2008)

26. Girardi, E. \& Zajonc, D. M. Molecular basis of lipid antigen presentation by CD1d and recognition by natural killer T cells. Immunol. Rev. 250, 167-179 (2012).

27. Reantragoon, R. et al. Structural insight into MR1-mediated recognition of the mucosal associated invariant T cell receptor. J. Exp. Med. 209, 761-774 (2012).

28. Mikkelsen, T. S. et al. Genome of the marsupial Monodelphis domestica reveals innovation in non-coding sequences. Nature 447, 167-177 (2007).

29. Patel, O. et al. Recognition of CD1d-sulfatide mediated by a type II natural killer T cell antigen receptor. Nat. Immunol. 13, 857-863 (2012).

30. Girardi, E. et al. Type II natural killer T cells use features of both innate-like and conventional T cells to recognize sulfatide self antigens. Nat. Immunol. 13, 851-856 (2012).

31. Pellicci, D. G. et al. Recognition of [beta]-linked self glycolipids mediated by natural killer T cell antigen receptors. Nat. Immunol. 12, 827-833 (2011).

32. Wun, K. S. et al. A molecular basis for the exquisite Cd1d-restricted antigen specificity and functional responses of natural killer T cells. Immunity 34, 327-339 (2011).

33. Garboczi, D. N., Hung, D. T. \& Wiley, D. C. HLA-A2-peptide complexes: refolding and crystallization of molecules expressed in Escherichia coli and complexed with single antigenic peptides. Proc. Natl Acad. Sci. USA 89, 3429-3433 (1992)

34. Leslie, A. G. The integration of macromolecular diffraction data. Acta Crystallogr. D Biol. Crystallogr. 62, 48-57 (2006).

35. CCP4. The CCP4 suite: programs for protein crystallography. Acta Crystallogr. D Biol. Crystallogr. 50, 760-763 (1994).

36. Zwart, P. H. et al. Automated structure solution with the PHENIX suite. Methods Mol. Biol. 426, 419-435 (2008).

37. Schuttelkopf, A. W. \& van Aalten, D. M. F. PRODRG: a tool for highthroughput crystallography of protein-ligand complexes. Acta Crystallogr. Biol. Crystallogr. 60, 1355-1363 (2004).

38. Bricogne, G. et al. autoBUSTER, Version 1.6.0 (Global Phasing Ltd, Cambridge, UK, 2011)
39. DeLano, W. L. The PyMOL Molecular Graphics System. http://www.pymol.org (2002).

40. Kjer-Nielsen, L. et al. A structural basis for the selection of dominant alphabeta $\mathrm{T}$ cell receptors in antiviral immunity. Immunity 18, 53-64 (2003).

\section{Acknowledgements}

We thank the staff of the Monash crystallization facility and the Australian Synchrotron for assistance with crystallization and data collection, respectively. This research was supported by the National Health and Medical Research Council of Australia (NHMRC) O.P. was supported by an ARC Future Fellowship; T.B. was supported by a Pfizer Australia Research Fellowship; J.J.M. was supported by an NHMRC Career Development Fellowship; D.P.F. was supported by an NHMRC Senior Principal Research Fellowship and J.R. was supported by an NHMRC Australia Fellowship.

\section{Author contributions}

J.L.N., T.B., A.J.C., L.L., J.J.M., B.M., R.R., M.L.S.-R., L.C.S., A.G.B., Z.C., R.B., S.B.G.E. and D.P.F. either performed the experiments, provided key reagents, and/or analysed the data, and/or provided intellectual input, or helped write the manuscript. J.McC. and J.R. co-led the investigation and contributed to the design and interpretation of data, project management and writing of the manuscript. J.McC. and J.R. are the joint senior and corresponding authors.

\section{Additional information}

Author Information: The atomic coordinates and structure factors for the TCR-MR1Ag complexes were deposited in the Protein Data Bank (PDB codes 4L4V and 4L4T).

Supplementary Information accompanies this paper at http://www.nature.com/ naturecommunications

Competing financial interests: The authors declare no competing financial interests.

Reprints and permission information is available online at http://npg.nature.com/ reprintsandpermissions/

How to cite this article: Patel, O. et al. Recognition of vitamin B metabolites by mucosalassociated invariant T cells. Nat. Commun. 4:2142 doi: 10.1038/ncomms3142 (2013). 